\title{
Invariant Tori of Nonlinear Schrödinger Equations with A Given Potential
}

\author{
Lijun Du and Xiaoping Yuan
}

\begin{abstract}
It is proved that for a given and analytic potential $V$ the nonlinear Schrödinger equation subject to Dirichlet boundary conditions possesses many elliptic invariant tori which carry quasi-periodic motions of high mode.
\end{abstract}

\section{Introduction and main results}

In this paper we deal with the existence of the invariant tori of the nonlinear Schrödinger equation

$$
\sqrt{-1} u_{t}=u_{x x}-V(x) u-f\left(|u|^{2}\right) u
$$

on the finite $x$-internal $[0, \pi]$ with Dirichlet boundary conditions

$$
u(t, 0)=0=u(t, \pi),-\infty<t<+\infty,
$$

where the potential $V$ is analytic in the strip domain $|\Im x|<r$ with some $r>0$, and $f$ is required to be real analytic in some neighborhood of the origin in $\mathbb{C}$. If $u=u(t, x)$ is a solution of equation (1), then, for any $c \in \mathbb{R}$, function $u(t, x) e^{i c t}$ solves

$$
\sqrt{-1} u_{t}=u_{x x}-(V(x)+c) u-f\left(|u|^{2}\right) u,
$$

with the boundary condition (2). Therefore we can assume that $\int_{0}^{\pi} V(x) d x=0$, and $f(0)=0$, by absorbing a constant into the constant $c$. Furthermore we require $f$ to be nondegenerate in the sense that $f^{\prime}(0) \neq 0$. As we will see later, the sign of the derivative of $f$ is immaterial for our results and may be assumed to be positive for convenience. Then we have

$$
\sqrt{-1} u_{t}=u_{x x}-V(x) u-|u|^{2} u+O\left(u^{5}\right) .
$$

after rescaling $u$ appropriately.

Historically, the investigations into the existence of time-quasi-periodic solutions for nonlinear partial differential equations were started independently by Kuksin $[\mathbf{2}]$ and Wayne $[\mathbf{8}]$. The reader is referred to Kuksin's monograph $[\mathbf{3}]$ for more details about the history. In the monograph, assuming $V=V(x, \sigma)$ depends

1991 Mathematics Subject Classification. 37, 34, 35, 78, 76.

Key words and phrases. Invariant tori, nonlinear Schrödinger equation, Dirichlet boundary condition, quasi-periodic motion.

This work was supported by NNSFC-10231020 and NCET-04-0365. 
on $n$-parameters $\sigma \in \mathbb{R}^{n}$, Kuksin showed that the equation (1) with b.c.(2) possesses many invariant tori for "most" (in the sense of Lebesgue measure) parameters $\sigma$. However, one does not know if there is any invariant torus of (1) for a given potential $V$, say, $V=\sin x$ or $V \equiv$ constant. There was a breakthrough in 1995: Kuksin and Pöschel [4] showed that for the equation (1) with $V(x) \equiv m$ there were many elliptic invariant tori which were the closure of some quasi-periodic solutions of the equation, where $m \in \mathbb{R}$ is a given constant. In the present paper, using some ideas and lemmas from $[\mathbf{9}]$, we shall show that there are many elliptic invariant tori of $(1)+(2)$ for a given analytic potential $V(x)$ which is not necessary to be constant. The technical heart of the present paper is the "separation" Lemma 2.4, while the rest is routine.

Following [4], we study this equation as a hamiltonian system on some suitable phase space $\mathcal{P}$, such as $\mathcal{P}=H_{0}^{1}([0, \pi])$, the Sobolev space of all complex valued $L^{2}$-functions on $[0, \pi]$ with an $L^{2}$-derivative and vanishing boundary values. With the inner product

and the Hamiltonian

$$
\langle u, v\rangle=\operatorname{Re} \int_{0}^{\pi} u \bar{v} d x
$$

$$
H=\frac{1}{2}\langle A u, u\rangle+\frac{1}{2} \int_{0}^{\pi} g\left(|u|^{2}\right) d x,
$$

where $A=-d^{2} / d x^{2}+V(x)$ and $g=\int_{0} f d z$, equation (1) can be written in the hamiltonian form

$$
u_{t}=i \nabla H(u)
$$

where the gradient of $H$ is defined with respect to $\langle\cdot, \cdot \cdot\rangle$.

Our aim is to construct plenty of small amplitude solution that are quasiperiodic in time. Such quasi-periodic solutions can be written in the form

$$
u(t, x)=U\left(\omega_{1} t, \cdots, \omega_{n} t, x\right),
$$

where $\omega_{1}, \cdots, \omega_{n}$ are rationally independent real numbers, the basic frequencies of $u$, and $U$ is a continuous function of period $2 \pi$ in the first $n$ arguments. Thus, $u$ admits a Fourier series expansion

$$
u(t, x) \sim \sum_{k \in \mathbf{Z}^{n}} U_{k}(x) e^{\sqrt{-1}\langle k, w\rangle t},
$$

where $\langle k, \omega\rangle=\sum_{j} k_{j} \omega_{j}$. We achieve our aim by constructing the $U$ as embeddings

$$
U: \mathbf{T} \rightarrow \mathcal{P}, \theta \mapsto U(\theta, \cdot)
$$

of the n-torus $\mathbf{T}^{n}$ into the phase space $\mathcal{P}$ together with frequency vectors $\omega$ such that the straight windings $t \mapsto \omega t+\theta_{0}$ on the torus map into solutions of equation (1).

Since the quasi-periodic solutions to be constructed are of small amplitude, the equation (1) may be considered as the linear equation $\sqrt{-1} u_{t}=u_{x x}-V(x) u$ with a small nonlinear perturbation $f\left(|u|^{2}\right) u$. Let $\phi_{j}(x)$ and $\lambda_{j}(j=1,2, \cdots)$ be the basic modes and their frequencies for the linear equation with Dirichlet boundary conditions. Then every solution of the linear system will be the superposition of oscillation of the basic modes, with the coefficients moving on circles:

$$
u(t, x)=\sum_{j \geq 1} q_{j}(t) \phi_{j}(x), q_{j}(t)=q_{j}^{0} e^{\sqrt{-1} \lambda_{j} t} .
$$


Together they move on a rotational torus of finite or infinite dimension, depending on how many modes are excited.

In particular, for every choice

$$
J=\left\{j_{1}<j_{2}<\cdots<j_{n}\right\} \subset \mathbb{N}
$$

of $n \geq 1$ basic modes there is an invariant linear space $E_{J}$ of complex dimension $n$ which is completely foliated into rotational tori:

$$
E_{J}=\left\{u=q_{1} \phi_{j_{1}}+\cdots+q_{n} \phi_{j_{n}}: q \in \mathbb{C}^{n}\right\}=\bigcup_{I \in \overline{\mathbf{P}^{n}}} \mathcal{T}_{J}(I),
$$

where $\mathbf{P}^{n}=\left\{I: I_{j}>0\right.$ for $\left.1 \leq j \leq n\right\}$ is the positive quadrant in $\mathbb{R}^{n}$ and

$$
\mathcal{T}_{J}(I)=\left\{u=q_{1} \phi_{j_{1}}+\cdots+q_{n} \phi_{j_{n}}:\left|q_{j}\right|^{2}=2 I_{j} \text { for } 1 \leq j \leq n\right\} .
$$

In addition, each such torus is linearly stable, and all solutions have vanishing Lyapunov exponents.

Upon restoration of the nonlinearity $f$, the invariant manifolds $E_{J}$ will not persist in their entirety due to resonances among the modes and the strong perturbing effect of $f$ for large amplitudes. We show, however, that in a sufficiently small neighborhood of the origin, a large Cantor subfamily of rotational $n$-tori persists and is only slightly deformed. That is, there exists a Cantor set $\mathcal{C} \subset \mathbf{P}^{n}$, a family of $n$-tori

$$
\mathcal{T}_{J}[\mathcal{C}]=\bigcup_{I \in \mathcal{C}} \mathcal{T}_{J}(I) \subset E_{J}
$$

over $\mathcal{C}$, and a Lipschitz continuous embedding

$$
\Psi: \mathcal{T}_{J}[\mathcal{C}] \hookrightarrow \mathcal{P},
$$

such that the restriction of $\Psi$ to each $\mathcal{T}_{J}(I)$ in the family is an embedding of a rotational $n$-torus for the nonlinear equation. The image $\mathcal{E}_{J}$ of $\mathcal{T}_{J}[\mathcal{C}]$ we call a Cantor manifold of rotational $n$-tori given by the embedding $\Psi: \mathcal{T}_{J}[\mathcal{C}] \rightarrow \mathcal{E}_{J}$.

THEOREM 1. Arbitrarily fix $n \in \mathbb{N}$. Assume that $V(x)$ is analytic in the strip domain $|\Im x|<r$ with $r>0$ and that

$$
\bar{V}:=\frac{1}{4 \pi}\left(V^{\prime}(0)-V^{\prime}(\pi)+\int_{0}^{\pi} V^{2}(x) d x\right) \neq 0 .
$$

Assume further that the nonlinearity $f$ is real analytic and nondegenerate. Then we can find many index sets $J=\left\{j_{1}<\cdots<j_{n}\right\}$ with $j_{1}$ large $^{1}$ enough to confirm that there exists a Cantor manifold $\mathcal{E}_{J}$ of real analytic, linearly stable, Diophantine $n$-tori for equation (1) given by a Lipschitz continuous embedding $\Psi: \mathcal{T}_{J}[\mathcal{C}] \rightarrow \mathcal{E}_{J}$, which is a higher order perturbation of the inclusion map $\Psi_{0}: E_{J} \hookrightarrow \mathcal{P}$ restricted to $\mathcal{T}_{J}[\mathcal{C}]$. The Cantor set $\mathcal{C}$ has full density at the origin, whence $\mathcal{E}_{J}$ is tangent to $E_{J}$ at the origin. In addition, the Diophantine tori carry quasi-periodic motions of high mode.

\footnotetext{
${ }^{1}$ The condition that $j_{1}$ is large enough is not necessary in [4].
} 


\section{The spectra of Sturm-Liouville problems}

Consider the Sturm-Liouville problems

$$
\left\{\begin{array}{c}
-\frac{d^{2} y}{d x^{2}}+V(x) y=\lambda y \\
y(0)=y(\pi)=0
\end{array}\right.
$$

In addition to assuming that $V(x)$ is analytic in a strip domain $|\Im x|<r$ with $r>0$, it is well known that the S-L problems possess infinitely many strictly increasing simple eigenvalues

$$
\lambda_{1}<\lambda_{2}<\cdots<\lambda_{\nu}<\cdots \rightarrow+\infty,
$$

and normalized eigenfunction $\phi_{\nu}$ corresponding to $\lambda_{\nu}$.

Lemma 2.1. Assume that $\int_{0}^{\pi} V(x) d x=0$. For the eigenvalues $\left\{\lambda_{\nu}\right\}$ and eigenfunction $\left\{\phi_{\nu}\right\}$ we have the following asymptotic formulas,

$$
\begin{gathered}
\lambda_{\nu}=\nu^{2}+\bar{V}\left(\frac{1}{\nu^{2}}\right)+O\left(\frac{1}{\nu^{3}}\right), \\
\phi_{\nu}(x)=\kappa_{\nu}^{-1}\left(\sin (\nu x)-\frac{\cos (\nu x)}{2 \nu} \int_{0}^{x} V(x) d s+\tilde{\phi}_{\nu}(x)\right),
\end{gathered}
$$

where $\kappa_{\nu}>0$ is a constant depending on $\nu$ such that $\left\|\phi_{\nu}\right\|_{L^{2}[0, \pi]}=1$, and

$$
\tilde{\phi}_{\nu}(x)=O\left(\frac{1}{\nu^{2}}\right), \tilde{\phi}_{\nu}^{\prime}(x)=O\left(\frac{1}{\nu}\right), \tilde{\phi}_{\nu}^{\prime \prime}(x)=O(1),
$$

uniformly for $x \in[0, \pi]$, and

$$
\bar{V}=\frac{1}{4 \pi}\left(V^{\prime}(0)-V^{\prime}(\pi)+\int_{0}^{\pi} V^{2}(x) d x\right) .
$$

Proof. The proofs are elementary, see $[\mathbf{6}]$ and $[\mathbf{7}]$ for details.

In the following argument, we assume $\bar{V} \neq 0$.

Lemma $2.2([\mathbf{9}])$. For $\kappa_{\nu}$ we have the following estimate:

$$
\kappa_{\nu}^{2}=\frac{\pi}{2}+O\left(\frac{1}{\nu^{2}}\right) .
$$

Lemma $2.3([\mathbf{9}])$. With respect to $\phi_{\nu}$ and $\phi_{\nu}^{\prime}$ we have that

$$
\kappa_{\nu}^{2} \kappa_{\nu^{\prime}}^{2} \int_{0}^{\pi} \phi_{\nu}^{2}(x) \phi_{\nu^{\prime}}^{2}(x) d x= \begin{cases}\frac{\kappa_{\nu^{\prime}}^{2}}{2}+O\left(\frac{1}{\nu^{2}}\right)+O\left(\frac{1}{\nu \nu^{\prime}\left|\nu-\nu^{\prime}\right|}\right), & \nu \neq \nu^{\prime}, \\ \frac{\kappa_{\nu}^{2}}{2}+\frac{\pi}{8}+O\left(\frac{1}{\nu^{2}}\right), & \nu=\nu^{\prime} .\end{cases}
$$

Proof. The proofs of the above lemmas can be found in [9].

LEMMA 2.4. Let $M$ be a positive integer large enough and let $N$ be an integer with $N \geq M+n$ where $n$ is the integer mentioned in Theorem 1. For any $i, j, k, l \in$ $\mathbb{N}$, if one of them is in $\{M, M+1, \cdots, N\}$, then we have

$$
\left|\lambda_{i}+\lambda_{j}-\lambda_{k}-\lambda_{l}\right|>\eta
$$

unless $\{i, j\}=\{k, l\}$, where $\eta$ is a constant depending on $V, M$ and $N$.

Proof. We should note that there two plus signs and two minus signs in the left of (11). This enables us to assume $i \geq j$ and $k \geq l$ without loss of generality. Set

$$
|\Upsilon|=\left|\lambda_{i}+\lambda_{j}-\lambda_{k}-\lambda_{l}\right|
$$


Case 1. If $\{i, j\} \cap\{k, l\} \neq \emptyset$, then $\Upsilon$ can be reduced to $|\Upsilon|=\left|\lambda_{i}-\lambda_{k}\right|$ with some $i \neq k$, by noting that the case $\{i, j\}=\{k, l\}$ is excluded in the assumption of this lemma. In this case, we can assume $i>k$ without loss of generality. Using the asymptotic formula (6), we get that there is a constant $C$ depending only on $V$ such that

$$
\begin{aligned}
|\Upsilon| & \geq \lambda_{i}-\lambda_{k} \\
& \geq i^{2}-k^{2}+\bar{V}\left(\frac{1}{i^{2}}-\frac{1}{k^{2}}\right)-\left|O\left(\frac{1}{k^{3}}\right)\right| \\
& \geq i^{2}-k^{2}-C \\
& >i+k-C .
\end{aligned}
$$

Therefore, if $i \geq C$, then $|\Upsilon| \geq k \geq 1$. If $i<C$, then

$$
|\Upsilon| \geq \inf _{k<i \leq C}\left\{\lambda_{i}-\lambda_{k}\right\}:=\eta_{0}>0 .
$$

Case 2. We assume $\{i, j\} \cap\{k, l\}=\emptyset$. This case can be reduced to the following two subcases: Case 2.1. $i \geq j>k \geq l$ (or $k \geq l>i \geq j$ ); Case 2.2. $i>k \geq l>j$ (or $k>i \geq j>l$ ).

Let us consider Case 2.1. In this case, we have

$$
|\Upsilon|=\left|\lambda_{i}+\lambda_{j}-\lambda_{k}-\lambda_{l}\right| \geq\left|\lambda_{i}-\lambda_{k}\right| .
$$

By the same argument as in Case 1 , we get $|\Upsilon| \geq \min \left\{1, \eta_{0}\right\}>0$. Finally, let us consider Case 2.2: $i>k \geq l>j$. Using (6), we have

$$
\begin{aligned}
|\Upsilon| & =\left|\lambda_{i}+\lambda_{j}-\lambda_{k}-\lambda_{l}\right| \\
& =\left|i^{2}+j^{2}-k^{2}-l^{2}+\bar{V}\left(\frac{1}{i^{2}}+\frac{1}{j^{2}}-\frac{1}{k^{2}}-\frac{1}{l^{2}}\right)+O\left(\frac{1}{j^{3}}\right)\right| \\
& >\left|i^{2}+j^{2}-k^{2}-l^{2}\right|-\left|\bar{V}\left(\frac{1}{i^{2}}+\frac{1}{j^{2}}-\frac{1}{k^{2}}-\frac{1}{l^{2}}\right)+O\left(\frac{1}{j^{3}}\right)\right| .
\end{aligned}
$$

It is clear that there is a constant $C_{1}$ large enough such that, for $j>C_{1}$,

$$
\left|\bar{V}\left(\frac{1}{i^{2}}+\frac{1}{j^{2}}-\frac{1}{k^{2}}-\frac{1}{l^{2}}\right)+O\left(\frac{1}{j^{3}}\right)\right| \leq 1 / 2 .
$$

Thus, we have Case 2.2.1: If $j>C_{1}$ and $i^{2}+j^{2} \neq k^{2}+l^{2}$, then

$$
|\Upsilon|>\left|i^{2}+j^{2}-k^{2}-l^{2}\right|-\frac{1}{2} \geq 1 / 2
$$

Case 2.2.2. Assume $j>C_{1}$ and $i^{2}+j^{2}=k^{2}+l^{2}$. (Remark. In [4], this case does not appear.) In this case, we set $i^{2}-k^{2}=l^{2}-j^{2}:=p>1$ and and let $q:=k^{2}-l^{2} \geq 0$. We substitute $\lambda_{\nu}$ in $\Upsilon$ with the asymptotic formula

$$
\lambda_{\nu}=\nu^{2}+\frac{\bar{V}}{\nu^{2}}+\frac{v_{3}}{\nu^{3}}+\frac{v_{4}}{\nu^{4}}+\frac{v_{5}}{\nu^{5}}+\frac{v_{6}}{\nu^{6}}+O\left(\frac{1}{\nu^{7}}\right),
$$


then

$$
\begin{aligned}
\Upsilon & =\bar{V}\left(\frac{1}{i^{2}}+\frac{1}{j^{2}}-\frac{1}{k^{2}}-\frac{1}{l^{2}}+\sum_{m=3}^{6} \frac{v_{m}}{\bar{V}}\left(\frac{1}{i^{m}}+\frac{1}{j^{m}}-\frac{1}{k^{m}}-\frac{1}{l^{m}}\right)\right)+O\left(\frac{1}{j^{7}}\right) \\
& =\bar{V}\left(\frac{1}{j^{2}}-\frac{1}{j^{2}+p}-\left(\frac{1}{j^{2}+p+q}-\frac{1}{j^{2}+p+p+q}\right)+\right. \\
& \left.\sum_{m=3}^{6} \frac{v_{m}}{\bar{V}}\left(\frac{1}{\left(j^{2}\right)^{\frac{m}{2}}}-\frac{1}{\left(j^{2}+p\right)^{\frac{m}{2}}}-\left(\frac{1}{\left(j^{2}+p+q\right)^{\frac{m}{2}}}-\frac{1}{\left(j^{2}+p+p+q\right)^{\frac{m}{2}}}\right)\right)\right) \\
& +O\left(j^{-7}\right) .
\end{aligned}
$$

Let

$$
f(t):=\frac{1}{j^{2}+t}-\frac{1}{j^{2}+p+t}+\sum_{m=3}^{6} \frac{v_{m}}{\bar{V}}\left(\frac{1}{\left(j^{2}+t\right)^{\frac{m}{2}}}-\frac{1}{\left(j^{2}+p+t\right)^{\frac{m}{2}}}\right) .
$$

It is easy to verify that for $t \geq 0$

$$
f^{\prime}(t)=\frac{-p\left(2 j^{2}+p+2 t\right)}{\left(j^{2}+t\right)^{2}\left(j^{2}+t+p\right)^{2}}\left(1+O\left(\frac{1}{\left(j^{2}+t\right)^{\frac{1}{2}}}\right)\right)<0,
$$

where we have chosen $C_{1}$ large enough such that

$$
\left|O\left(\frac{1}{\left(j^{2}+t\right)^{\frac{1}{2}}}\right)\right| \leq 1 / 2, \text { for } j>C_{1} .
$$

This implies that

$$
\begin{aligned}
& f(0)-f(p+q) \geq f(0)-f(p) \\
& =\frac{1}{j^{2}}+\frac{1}{j^{2}+2 p}-\frac{2}{j^{2}+p}+\sum_{m=3}^{6} \frac{v_{m}}{\bar{V}}\left(\frac{1}{\left(j^{2}\right)^{\frac{m}{2}}}+\frac{1}{\left(j^{2}+2 p\right)^{\frac{m}{2}}}-\frac{2}{\left(j^{2}+p\right)^{\frac{m}{2}}}\right) \\
& :=g(p) .
\end{aligned}
$$

Replacing $p$ in $g(p)$ by $t \in[0,+\infty)$, we get a function $g(t)$. Then

$$
g^{\prime}(t)=\frac{4 t j^{2}+6 t^{2}}{\left(j^{2}+2 t\right)^{2}\left(j^{2}+t\right)^{2}}\left(1+O\left(\frac{1}{\left(j^{2}+t\right)^{\frac{1}{2}}}\right)\right)>0, \text { for } t>0 .
$$

It follows from $p>1$ that $g(p)>g(1)$.

Thus,

$$
\begin{aligned}
& f(0)-f(p+q) \geq f(0)-f(p)=g(p)>g(1) \\
& =\frac{2}{j^{2}\left(j^{2}+1\right)\left(j^{2}+2\right)}+\sum_{m=3}^{6} \frac{v_{m}}{\bar{V}}\left(\frac{1}{\left(j^{2}\right)^{\frac{m}{2}}}+\frac{1}{\left(j^{2}+2\right)^{\frac{m}{2}}}-\frac{2}{\left(j^{2}+1\right)^{\frac{m}{2}}}\right) .
\end{aligned}
$$

Then we have

$$
\begin{aligned}
|\Upsilon| & =\left|\bar{V}(f(0)-f(p+q))+O\left(\frac{1}{j^{7}}\right)\right| \\
& >\frac{2|\bar{V}|}{j^{2}\left(j^{2}+1\right)\left(j^{2}+2\right)}\left(1+O\left(\frac{1}{\left(j^{2}+1\right)^{\frac{1}{2}}}\right)\right)+O\left(\frac{1}{j^{7}}\right) .
\end{aligned}
$$

Therefore, when $j>C_{1} \gg 1$, we have

$$
|\Upsilon|>\frac{|\bar{V}|}{2 j^{2}\left(j^{2}+1\right)\left(j^{2}+2\right)} \text {. }
$$


Since one of $i, j, k, l$ is in the set $\{M, M+1, \ldots, N\}$, we have $j \leq N$. Thus,

$$
|\Upsilon| \geq \frac{|\bar{V}|}{2\left(N^{2}+2\right)^{3}}
$$

Case 2.2.3. $j<C_{1}$ and $l \leq k<\sqrt{M}$. Note the constant $C_{1} \gg 1$ is independent of $M$. Let $M \geq C_{1}^{6}$. In this case, since one of $i, j, k, l$ is in the set $\{M, M+1, \ldots, N\}$, we have $i \geq M$. We have

$$
\lambda_{i}=i^{2}+\frac{\bar{V}}{i^{2}}+O\left(\frac{1}{i^{3}}\right)>M^{2}-\frac{1}{2}>\left|\lambda_{k}\right|+\left|\lambda_{l}\right|+\left|\lambda_{j}\right|+1 .
$$

Hence $|\Upsilon|>1$.

Case 2.2.4. $j<C_{1}$ and $l<\sqrt{M} \leq k$. In this case, since one of $i, j, k, l$ is in the set $\{M, M+1, \ldots, N\}$, we still have $i \geq M$. Recall $i>k$. Thus, $i-k \geq 1$. Moreover,

$\lambda_{i}-\lambda_{k}=i^{2}-k^{2}-\frac{\bar{V}}{k^{2}}+\frac{\bar{V}}{i^{2}}+O\left(\frac{1}{k^{3}}\right)>i+k-\frac{1}{2} \geq M+\sqrt{M}-\frac{1}{2}>\left|\lambda_{l}\right|+\left|\lambda_{j}\right|+1$ where we have used $M \gg C_{1}$ and $C_{1} \gg 1$. So $|\Upsilon|>1$.

Case 2.2.5. $j<C_{1}$ and $l \geq \sqrt{M}$. In this case, $l \leq N$. If $\lambda_{j}+i^{2}-k^{2}-l^{2}=0$, we have

$$
|\Upsilon|=\left|\frac{\bar{V}}{i^{2}}-\frac{\bar{V}}{k^{2}}-\frac{\bar{V}}{l^{2}}+O\left(\frac{1}{l^{3}}\right)\right|>\frac{|\bar{V}|}{2 l^{2}}>\frac{|\bar{V}|}{2 N^{2}} .
$$

If $\lambda_{j}+i^{2}-k^{2}-l^{2} \neq 0$, let

$$
\eta_{1}=\eta_{1}\left(C_{1}\right)=\inf _{j<C_{1}}\left\{\left|\lambda_{j}+i^{2}-k^{2}-l^{2}\right|: \lambda_{j}+i^{2}-k^{2}-l^{2} \neq 0\right\}>0,
$$

we have

$$
\begin{aligned}
|\Upsilon| & \geq\left|\lambda_{j}+i^{2}-k^{2}-l^{2}\right|-\left|\frac{\bar{V}}{i^{2}}-\frac{\bar{V}}{k^{2}}-\frac{\bar{V}}{l^{2}}+O\left(\frac{1}{l^{3}}\right)\right| \\
& >\eta_{1}-2\left|\frac{\bar{V}}{M}+O\left(M^{-3 / 2}\right)\right| \\
& >\eta_{1}-\frac{\eta_{1}}{2}=\frac{\eta_{1}}{2}
\end{aligned}
$$

provided that $M>10 \bar{V} / \eta_{1}$ and $M \gg C_{1}$. Finally, let

$$
\eta=\min \left\{\frac{|\bar{V}|}{2\left(N^{2}+2\right)^{3}}, \eta_{0}, \frac{\eta_{1}}{2}, \frac{1}{2}\right\},
$$

we complete the proof.

\section{The Hamiltonian}

The Hamiltonian of the nonlinear Schrödinger equation is

$$
H=\frac{1}{2}\langle A u, u\rangle+\frac{1}{2} \int_{0}^{\pi} g\left(|u|^{2}\right) d x,
$$

where $A=-d^{2} / d x^{2}+V(x)$ and $g=\int_{0} f d z$. We rewrite $H$ as a Hamiltonian in infinitely many coordinates by making the transformation

$$
u=\mathcal{S} q=\sum_{j \geq 1} q_{j} \phi_{j}
$$


where $\phi_{j}$ are the eigenfunctions of the Sturm-Liouville problems mentioned above. The coordinates are taken from the Hilbert space $\ell^{a, p}$ of all complex-valued sequence $q=\left(q_{1}, q_{2}, \ldots\right)$ with

$$
\|q\|_{a, p}^{2}=\sum_{j \geq 1}\left|q_{j}\right|^{2} j^{2 p} e^{2 j a}<\infty
$$

where $a>0$ and $p>\frac{1}{2}$ will be fixed later. We then obtain the Hamiltonian

$$
H=\Lambda+G=\frac{1}{2} \sum_{j \geq 1} \lambda_{j}\left|q_{j}\right|^{2}+\frac{1}{2} \int_{0}^{\pi} g\left(|\mathcal{S} q|^{2}\right) d x
$$

on the space $\ell^{a, p}$ with symplectic structure $\sqrt{-1} \sum_{j} d q_{j} \wedge d \bar{q}_{j}$. The Hamiltonian equation is

$$
\dot{q}_{j}=2 \sqrt{-1} \frac{\partial H}{\partial \bar{q}_{j}}, j \geq 1 .
$$

LEMMA 3.1. Let $a>0$ and $p \geq 1 / 2$. If a curse $I \rightarrow \ell^{a, p}, t \mapsto q(t)$ is an analytic solution of (14), then

$$
u(t, x)=\sum_{j \geq 1} q_{j}(t) \phi_{j}(x)
$$

is a solution of (1) that is analytic on $I \times[0, \pi]$.

The proof can be found in $[\mathbf{4}]$.

Let $\ell_{b}^{2}$ and $L^{2}$, respectively, be the Hilbert spaces of all bi-infinite, square summable sequences with complex coefficients and all square-integrable complexvalued functions on $[-\pi, \pi]$. Let

$$
\mathcal{F}: \ell_{b}^{2} \rightarrow L^{2}, q \mapsto \mathcal{F} q=\frac{1}{\sqrt{2 \pi}} \sum_{j \in \mathbb{Z}} q_{j} e^{\sqrt{-1} j x}
$$

be the inverse discrete Fourier transform, which defines an isometry between the two spaces. Let $a \geq 0$ and $p \geq 1 / 2$. The subspace $\ell_{b}^{a, p} \subset \ell_{b}^{2}$ consists, by definition, of all bi-infinite sequences with finite form

$$
\|q\|_{a, p}^{2}=\left|q_{0}\right|^{2}+\sum_{j \in \mathbb{Z}}\left|q_{j}\right|^{2}|j|^{2 p} e^{2|j| a} .
$$

Let $H^{a, p}:=\mathcal{F}\left(\ell^{a, p}\right)$. Then $H^{a, p} \subset L^{2}$. Set

$$
\|\mathcal{F} q\|_{a, p}=\|q\|_{a, p} .
$$

For $a>0$, the spaces $H^{a, p}$ may be identified with the spaces of all $2 \pi$-periodic functions which are analytic and bounded in the complex strip $|\Im z|<a$ with trace functions on $|\Im z|=a$ belonging to the usual Sobolev space $H^{p}$.

LEMma 3.2. For $a \geq 0$ and $p>\frac{1}{2}$, the space $\ell_{b}^{a, p}$ is a Hilbert algebra with respect to convolution of sequences, and

$$
\left\|q * q^{\prime}\right\|_{a, p} \leq h\|q\|_{a, p}\left\|q^{\prime}\right\|_{a, p}
$$

with a constant $h$ depending only on $p$. Consequently, $H^{a, p}$ is a Hilbert algebra with respect to multiplication of functions.

LEMMA 3.3. For $a \geq 0$ and $p>\frac{1}{2}$, the hamiltonian vectorfield $\mathbf{X}_{G}$ is a real analytic as a map from some neighborhood of the origin in $\ell^{a, p}$ into $\ell^{a, p}$, with

$$
\left\|\mathbf{X}_{G}\right\|_{a, p}=O\left(\|q\|_{a, p}^{3}\right) .
$$


The proofs of Lemmas 3.2 and 3.3 can be found in [4]. Thus $\mathbf{X}_{G}$ is a genuine vectorfield on $\ell^{a, p}$. On the other hand the linear vectorfield $\mathbf{X}_{\Lambda}$ is unbounded on $\ell^{a, p}$, since it takes values in $\ell^{a, p-2}$.

For the nonlinearity $|u|^{2} u$ we find

$$
G=\frac{1}{4} \int_{0}^{\pi}|u(x)|^{4} d x=\frac{1}{4} \sum_{i, j, k, l} G_{i j k l} q_{i} q_{j} \bar{q}_{k} \bar{q}_{l}
$$

with

In particular, we have

$$
G_{i j k l}=\int_{0}^{\pi} \phi_{i} \phi_{j} \phi_{k} \phi_{l} d x
$$

$$
\kappa_{i}^{2} \kappa_{j}^{2} G_{i j i j}=\kappa_{i}^{2} \kappa_{j}^{2} \int_{0}^{\pi} \phi_{i}^{2}(x) \phi_{j}^{2}(x) d x
$$

\section{Partial Birkhorff normal form}

Firstly let us introduce some notations. Set $\Xi:=\{M, M+1, \ldots, N\}$. For a vector $q \in \ell^{a, p}$, we write $q=\left(q_{j}: j \in \mathbb{Z}\right)$. Let $\tilde{q}=\left(q_{j}: j \in \Xi\right)$ and $\hat{q}=\left(q_{j}: j \in\right.$ $\mathbb{Z} \backslash \Xi)$. Then $q=\tilde{q} \oplus \hat{q}$. Define $\|\hat{q}\|_{a, p}:=\|\tilde{q} \oplus \hat{q}\|_{a, p}$ by replacing $\tilde{q}$ by 0 .

LEMMA 4.1. There exits a real analytic, symplectic change of coordinates $\Gamma$ in a neighborhood of the origin in $\ell^{a, p}$ such that the Hamiltonian $H=\Lambda+G$ with nonlinearity (15) is changed into a partial Birkhoff normal form up to order four, which reads

$$
H \circ \Gamma=\Lambda+\bar{G}+\widehat{G}+K,
$$

where $\mathbf{X}_{\bar{G}}, \mathbf{X}_{\widehat{G}}, \mathbf{X}_{K}$ are real analytic vectorfields in a neighborhood of the origin in $\ell^{a, p}$.

$$
\bar{G}=\frac{1}{2} \sum_{\text {one of }\{i, j\} \in \Xi} \bar{G}_{i j}\left|q_{i}\right|^{2}\left|q_{j}\right|^{2},
$$

with $\bar{G}_{i j}=G_{i j i j}$ and

$$
|\widehat{G}| \leq C\|\hat{q}\|_{a, p}^{4},|K| \leq C\|q\|_{a, p}^{6}
$$

where the constant $C$ depends on $M, N$ and $n$.

Proof. The proof is based on Lemma 2.4. Let $\Gamma=\left.\mathbf{X}_{F}^{t}\right|_{t=1}$ be the time-1-map of the flow of the hamiltonian vectorfield $\mathbf{X}_{F}$ given by the Hamiltonian

$$
F=\frac{1}{4} \sum_{i, j, k, l} F_{i j k l} q_{i} q_{j} \bar{q}_{k} \bar{q}_{l}
$$

with coefficients

$$
\sqrt{-1} F_{i j k l}=\left\{\begin{array}{cl}
\frac{G_{i j k l}}{\lambda_{i}+\lambda_{j}-\lambda_{k}-\lambda_{l}} & \text { for }(i, j, k, l) \in \mathcal{M} \backslash \mathcal{N}, \\
0 & \text { otherwise. }
\end{array}\right.
$$

where

$$
\mathcal{M}=\left\{(i, j, k, l) \in \mathbb{N}^{4}: \text { one of }\{i, j, k, l\} \in \Xi\right\},
$$

and

$$
\mathcal{N}=\{(i, j, k, l) \in \mathcal{M}:\{i, j\}=\{k, l\}\} .
$$

With the Lemma 2.4, we can see that each entry of $\mathbf{X}_{F}$ is a polynomial of degree 3 . And as in [1], we can show that $\mathbf{X}_{F}$ is a real analytic vector field in the neighborhood 
of the origin in $\ell^{a, p}$. Hence $\Gamma$ is a real analytic, symplectic change of coordinates defined at least in a neighborhood of the origin in $\ell^{a, p}$. Expanding $\left.H \circ \mathbf{X}_{F}^{t}\right|_{t=1}$ at $t=0$ by Taylor's formula we have

$$
\begin{aligned}
H \circ \Gamma= & \left.H \circ \mathbf{X}_{F}^{t}\right|_{t=1} \\
=H & +\{H, F\}+\int_{0}^{1}(1-t)\{\{H, F\}, F\} \circ \mathbf{X}_{F}^{t} d t \\
=\Lambda & +G+\{\Lambda, F\} \\
& +\{G, F\}+\int_{0}^{1}(1-t)\{\{H, F\}, F\} \circ \mathbf{X}_{F}^{t} d t
\end{aligned}
$$

where $\{\cdot, \cdot\}$ denotes the Poisson bracket with respect to the symplectic structure. By direct computation of $\{H, F\},\{\{H, F\}, F\}$ and $\{G, F\}$, the proof is finished. The details can be found in $[\mathbf{4}]$.

\section{The Cantor manifold theorem}

In this section, we recite the Cantor Manifold Theorem in Kuksin and Pöschel's paper [4]. In a neighborhood of the origin in $\ell^{a, p}$ we consider more generally Hamiltonian of the form $H=\Lambda+Q+R$, where $\Lambda+Q$ is integrable term and $R$ is perturbation term. More precisely, let $q=(\tilde{q}, \hat{q})$ with $\tilde{q}=\left(q_{1}, \cdots, q_{n}\right)$ and $\hat{q}=\left(q_{n+1}, q_{n+2}, \cdots\right)$, and let

$$
I=\frac{1}{2}\left(\left|q_{1}\right|^{2}, \cdots,\left|q_{n}\right|^{2}\right), Z=\frac{1}{2}\left(\left|q_{n+1}\right|^{2},\left|q_{n+2}\right|^{2}, \cdots\right) .
$$

Letting $\alpha$ be a $n$-dimensional vector, $\beta$ an infinite dimensional vector, and letting $A$ be a $n \times n$ matrix, $B$ an $\infty \times n$ matrix, we assume that

$$
\Lambda=\langle\alpha, I\rangle+\langle\beta, Z\rangle, Q=\frac{1}{2}\langle A I, I\rangle+\langle B I, Z\rangle,
$$

where $\langle\cdot, \cdot\rangle$ is usual real scalar product. The equations of motion of the Hamiltonian $\Lambda+Q$ are

$$
\begin{array}{lr}
\dot{\tilde{q}}_{i}=\sqrt{-1}\left(\alpha+A I+B^{T} Z\right)_{i} \tilde{q}_{i}, & 1 \leq i \leq n, \\
\dot{\hat{q}}_{j}=\sqrt{-1}(\beta+B I)_{j} \hat{q}_{j}, & j \geq 1,
\end{array}
$$

where $T$ means the transpose of the matrix. Thus, the complex n-dimensional manifold $E=\{\hat{q}=0\}$ is invariant, and it is completely filled up to the origin by the invariant tori

$$
\mathcal{T}(I)=\left\{\tilde{q}:\left|\tilde{q}_{i}\right|^{2}=2 I_{i} \text { for } 1 \leq i \leq n\right\}, I \in \overline{\mathbf{P}^{n}} .
$$

On $\mathcal{T}(I)$ the flow is given by the equations

$$
\dot{\tilde{q}}_{i}=\sqrt{-1} \omega_{i}(I) \tilde{q}_{i} \text {, here }, \omega_{i}(I)=(\alpha+A I)_{i},
$$

and in its normal space by

$$
\dot{\hat{q}}_{j}=\sqrt{-1} \Omega_{j}(I) \hat{q}_{j}, \text { here }, \Omega_{j}(I)=(\beta+B I)_{j} .
$$

Since $\Omega(I)$ is real, $\hat{q}=0$ is an elliptic fixed point, all the tori are linear stable, and all their orbits have zero Lyapunov exponents. We therefor call $\mathcal{T}(I)$ an elliptic rotational torus with frequencies $\omega(I)$.

Including the nonintegrable perturbation term $R$ this manifold $E$ does in general not persist in its entirety due to resonances among the oscillation. Instead, our 
aim is to prove the persistence of a large portion of $E$ forming an invariant Cantor manifold $\mathcal{E}$ for the Hamiltonian $H=\Lambda+Q+R$. That is, there exists a family of $n$-tori

$$
\mathcal{T}[\mathcal{C}]=\bigcup_{I \in \mathcal{C}} \mathcal{T}(I) \subset E
$$

over a Cantor set $\mathcal{C} \subset \mathbf{P}^{n}$ and a Lipschitz continuous embedding

$$
\Psi: \mathcal{T}[\mathcal{C}] \hookrightarrow \ell^{a, p},
$$

such that the restriction of $\Psi$ to each torus $\mathcal{T}(I)$ in the family is an embedding of an elliptic rotational $n$-torus for the Hamiltonian $H$. We call the image $\mathcal{E}$ of $\mathcal{T}(\mathcal{C})$ a Cantor manifold of elliptic rotational n-tori given by the embedding $\Psi: \mathcal{T}(\mathcal{C}) \rightarrow \mathcal{E}$.

In addition, the Cantor set $\mathcal{C}$ has full density at the origin, the embedding $\Psi$ is close to the inclusion map $\Psi_{0}: E \hookrightarrow \ell^{a, p}$, and the Cantor manifold $\mathcal{E}$ is tangent to $E$ at the origin.

For the existence of $\mathcal{E}$ the following assumptions are made.

A. Nondegeneracy. The normal form $\Lambda+Q$ is nondegenerate in the sense that

$$
\begin{gathered}
\operatorname{det} A \neq 0, \\
\langle l, \beta\rangle \neq 0, \\
\langle k, \omega(I)\rangle+\langle l, \Omega(I)\rangle \neq \equiv 0,
\end{gathered}
$$

for all $(k, l) \in \mathbb{Z}^{n} \times \mathbb{Z}^{\infty}$ with $1 \leq|l| \leq 2$, where $\omega=\alpha+A I$ and $\Omega=\beta+B I$.

B. Spectral Asymptotics. There exist $d \geq 1$ and $\delta<d-1$ such that

$$
\beta_{j}=j^{d}+\cdots+O\left(j^{\delta}\right),
$$

where the dots stands for terms of order less than $d$ in $j$.

C. Regularity.

$$
\mathbf{X}_{Q}, \mathbf{X}_{R} \in \mathcal{A}\left(\ell^{a, p}, \ell^{a, \bar{p}}\right), \begin{cases}\bar{p} \geq p & \text { for } d>1 \\ \bar{p}>p & \text { for } d=1,\end{cases}
$$

where $\mathcal{A}\left(\ell^{a, p}, \ell^{a, \bar{p}}\right)$ denotes the class of all maps from some neighborhood of the origin in $\ell^{a, p}$ into $\ell^{a, \bar{p}}$, which are real analytic in the real and imaginary parts of the complex coordinate $q$.

THEOREM 2 (The Cantor Manifold Theorem). Suppose the Hamiltonian $H=\Lambda+Q+R$ satisfies assumptions $A, B$ and $C$, and

$$
|R|=O\left(\|q\|_{a, p}^{g}\right)+O\left(\|\hat{q}\|_{a, p}^{4}\right)
$$

with

$$
g>4+\frac{4-\Delta}{\kappa}, \Delta=\min (\bar{p}-p, 1) .
$$

Then there exists a Cantor manifold $\mathcal{E}$ of real analytic, elliptic Diophantine $n$-tori given by a Lipschitz continuous embedding $\Psi: \mathcal{T}[\mathcal{C}] \rightarrow \mathcal{E}$, where $\mathcal{C}$ has full density at the origin, and $\Psi$ is close to the inclusion map $\Psi_{0}$ :

$$
\left\|\Psi-\Psi_{0}\right\|_{a, \bar{p}, B_{r} \cap \mathcal{T}[\mathcal{C}]}=O\left(r^{\sigma}\right), \sigma=\frac{g}{2}-\frac{\kappa+1-\Delta / 4}{\kappa}>1 .
$$

Consequently, $\mathcal{E}$ is tangent to $E$ at the origin.

The proof of the Cantor Manifold Theorem can be found in [4]. 


\section{Proof of the main theorem}

Now we want to apply Theorem 2 to the Hamiltonian $H \circ \Gamma$ defined in Lemma 4.1. Let $N=M+n$. Rewrite

$$
\Xi=\{M, M+1, \ldots, N\}:=\left\{j_{1}, \ldots, j_{n}\right\}:=J,
$$

where $M \gg 1$ is defined in Lemma 2.4. In Lemma 4.1, we have shown that there is a real analytic symplectic map $\Gamma$ such that the Hamiltonian $H$ defined in (13) is changed into a a partial Birkhorff normal form up to order four:

$$
H \circ \Gamma=\Lambda+\bar{G}+\hat{G}+K,
$$

where

$$
\bar{G}=\frac{1}{2} \sum_{\text {one of }\{i, j\} \in J} \bar{G}_{i j}\left|q_{i}\right|^{2}\left|q_{j}\right|^{2} .
$$

Let $q=(\tilde{q}, \hat{q})$ on $\ell^{a, p}$, where $\tilde{q}=\left(q_{j_{1}}, \cdots, q_{j_{n}}\right)$ and $\hat{q}=q \ominus \tilde{q}$. Then $\hat{G}=O\left(\|\hat{q}\|_{a, p}^{4}\right)$, $|K|=O\left(\|q\|_{a, p}^{6}\right)$.

With the notation of section 5 we can write

$$
\Lambda=\langle\alpha, I\rangle+\langle\beta, Z\rangle, \bar{G}=\frac{1}{2}\langle A I, I\rangle+\langle B I, Z\rangle,
$$

where $\alpha=\left(\lambda_{j}\right)_{j \in J}, \beta=\left(\lambda_{j}\right)_{j \notin J}$ and $A=\left(\bar{G}_{i j}\right)_{i, j \in J}, B=\left(\bar{G}_{j i}\right)_{i \in J, j \notin J}$. Thus the transformed Hamiltonian is of the form $H \circ \Gamma=\Lambda+Q+R$ with $Q=\bar{G}$ and $R=\hat{G}+K$, as required by the Cantor Manifold Theorem. Then we only need to verify the hypotheses of Theorem 2 .

Lemma 6.1. When $M \gg 1$, condition $A$ is fulfilled.

Proof. By (16) and Lemma 2.3 we have

$$
\begin{array}{ll}
\kappa_{i}^{2} \kappa_{j}^{2} \bar{G}_{i j}=\kappa_{i}^{2} \kappa_{j}^{2} G_{i j i j}=\frac{\kappa_{j}^{2}}{2}+O\left(\frac{1}{i^{2}}\right)+O\left(\frac{1}{i j|i-j|}\right) & i \neq j, \\
\kappa_{i}^{2} \kappa_{j}^{2} \bar{G}_{i j}=\frac{\kappa_{i}^{4}}{2} G_{i i i i}=\frac{\kappa_{i}^{2}}{4}+\frac{\pi}{16}+O\left(\frac{1}{i^{2}}\right) & i=j .
\end{array}
$$

And with $\kappa_{i}^{2}=\frac{\pi}{2}+O\left(\frac{1}{i^{2}}\right)$, we get

$$
\begin{array}{lr}
\bar{G}_{i j}=\frac{4-\delta_{i j}}{4 \pi}+O\left(\frac{1}{i^{2}}\right)+O\left(\frac{1}{j^{2}}\right)+O\left(\frac{1}{i j|i-j|}\right) & i, j \in J, \\
\bar{G}_{i j}=\frac{1}{\pi}+O\left(\frac{1}{i^{2}}\right)+O\left(\frac{1}{i j|i-j|}\right) & i \in J, j \notin J .
\end{array}
$$

Let $a_{i j}=\bar{G}_{i j}-\frac{4-\delta_{i j}}{4 \pi}$, there exist a constant $b$ independent of $M$, such that

$$
\begin{array}{rlr}
\left|a_{i j}\right| & \leq \frac{b}{M^{2}}, \\
\left|a_{i j}\right| & \leq \frac{b}{M}, & i, j \in J,
\end{array}
$$

Recall $A=\left(\bar{G}_{i j}\right)_{i, j \in J}$. We have $4 \pi A=4 X-I+\tilde{A}$, where $I$ is the identity matrix and all elements of $X$ are 1 , and $\tilde{A}=\left(a_{i j}\right)_{i, j \in J}$. For $\operatorname{det}(4 X-I) \neq 0$, we know that there exists an elementary transformation $T$, such that $T(4 X-I)=$ $\operatorname{diag}\left(\sigma_{1}, \sigma_{2}, \cdots, \sigma_{n}\right)$ and $\operatorname{det}(4 X-I)=\operatorname{det} T(4 X-I)$, where

$$
\sigma_{i}=-1-\frac{4}{3+4(i-2)} \text {. }
$$


Putting the same transformation on $4 X-I+\tilde{A}$ and letting $T \tilde{A}=\dot{\tilde{A}}$, we know that

$$
\operatorname{det}(4 X-I+\tilde{A})=\operatorname{det}\left(\operatorname{diag}\left(\sigma_{1}, \sigma_{2}, \cdots, \sigma_{n}\right)+\dot{\tilde{A}}\right) .
$$

Let $\dot{a}_{i j}$ are the elements of $\dot{\tilde{A}}$, by (17) we can choose $M$ large enough to make the matrix

$$
\left(\begin{array}{cccc}
\sigma_{1}+\dot{a}_{11} & \dot{a}_{12} & \cdots & \dot{a}_{1 n} \\
\dot{a}_{21} & \sigma_{2}+\dot{a}_{22} & \cdots & \dot{a}_{2 n} \\
\vdots & \vdots & \ddots & \vdots \\
\dot{a}_{n 1} & \dot{a}_{n 2} & \cdots & \sigma_{n}+\dot{a}_{n n}
\end{array}\right)
$$

satisfying $\left|\sigma_{j}\right|>\sum_{i=1}^{n}\left|\dot{a}_{i j}\right|$, therefor the determinant of this matrix doesn't be zero. So $\operatorname{det}(4 X-I+\tilde{A}) \neq 0$, that is $\operatorname{det} A \neq 0$.

Clearly, $\langle l, \beta\rangle \neq 0$ for $1 \leq|l| \leq 2$. In order to verify the nondegenerate condition A, we have to check that

$$
\langle\alpha, k\rangle+\langle\beta, l\rangle \neq 0 \text { or } A k+B^{T} l \neq 0
$$

for all $(k, l)$ with $1 \leq|l| \leq 2$. Write $l$ as

$$
l=\left(\cdots, l_{j}, \cdots, l_{j_{0}}, \cdots, l_{j_{0}^{\prime}}, \cdots\right),
$$

where $l_{j}=0$ for $j \notin\left\{j_{0}, j_{0}^{\prime}\right\}$, and $l_{j_{0}}, l_{j_{0}^{\prime}} \in\{0,-1,1\}$ with $\left|l_{j_{0}}\right|+\left|l_{j_{0}^{\prime}}\right| \neq 0$. Recall $B^{T}=\left(\bar{G}_{i j}\right)_{i \in J, j \notin J}$. Let $k=\left(k_{1}, \cdots, k_{s}, \cdots, k_{n}\right)$. Suppose

$$
A k+B^{T} l=0 .
$$

Multiplying both sides of the equation by $4 \pi$ we have,

$$
\sum_{t=1}^{n}\left(4+a_{i_{s} i_{t}}\right) k_{t}-k_{s}+\left(4+a_{i_{s} j_{0}}\right) l_{j_{0}}+\left(4+a_{i_{s} j_{0}^{\prime}}\right) l_{j_{0}^{\prime}}=0, s=1,2, \cdots, n .
$$

Taking $s=s_{1}$ and $s=s_{2}$ in (19), we get two equations (19- $\left.s_{1}\right)$ and (19- $\left.s_{2}\right)$. Considering (19- $\left.s_{1}\right)$ minus $\left(19-s_{2}\right)$, we get

(20) $\sum_{t=1}^{n}\left(a_{i_{s_{1}} i_{t}}-a_{i_{s_{2}} i_{t}}\right) k_{t}+k_{s_{2}}-k_{s_{1}}+\left(a_{i_{s_{1}} j_{0}}-a_{i_{s_{2}} j_{0}}\right) l_{j_{0}}+\left(a_{i_{s_{1}} j_{0}^{\prime}}-a_{i_{s_{2}} j_{0}^{\prime}}\right) l_{j_{0}^{\prime}}=0$.

Moreover, by (19), (20) and noting $i_{s}, i_{t} \in J$, we have

$$
\begin{aligned}
\left|k_{s_{2}}-k_{s_{1}}\right| & \leq\left|\sum_{t=1}^{n}\left(a_{i_{s_{1}} i_{t}}-a_{i_{s_{2}} i_{t}}\right) k_{t}\right|+\left|a_{i_{s_{1}} j_{0}}-a_{i_{s_{2}} j_{0}}\right|+\left|a_{i_{s_{1}} j_{0}^{\prime}}-a_{i_{s_{2}} j_{0}^{\prime}}\right| \\
& <\sum_{t=1}^{n}\left|a_{i_{s_{1}} i_{t}}-a_{i_{s_{2}} i_{t}}\right|\left|k_{t}\right|+\left|a_{i_{s_{1}} j_{0}}-a_{i_{s_{2}} j_{0}}\right|+\left|a_{i_{s_{1}} j_{0}^{\prime}}-a_{i_{s_{2}} j_{0}^{\prime}}\right| \\
& <\frac{2 b}{M^{2}} \sum_{t=1}^{n}\left|k_{t}\right|+\frac{4 b}{M} .
\end{aligned}
$$

Assume $\left|k_{s_{2}}-k_{s_{1}}\right|<1$. Then $k_{s_{2}}=k_{s_{1}}$, since $\mathrm{k}$ is an integer vector. Thus there is an integer $h$ such that all elements of $k$ are equal to $h$. Therefore we can rewrite the equations (19) as

$$
(4 n-1) h+h \sum_{t=1}^{n} a_{i_{s} i_{t}}+\left(4+a_{i_{s} j_{0}}\right) l_{j_{0}}+\left(4+a_{i_{s} j_{0}^{\prime}}\right) l_{j_{0}^{\prime}}=0 .
$$


By (19), (20) and $i_{s}, i_{t} \in J$, we can choose $M$ large enough, such that

$$
3\left|\sum_{t=1}^{n} a_{i_{s} i_{t}}\right|+\left|a_{i_{s} j_{0}}\right|+\left|a_{i_{s} j_{0}^{\prime}}\right|<\frac{3 n b}{M^{2}}+\frac{2 b}{M}<1 .
$$

Then

$$
h=\frac{\left(4+a_{i_{s} j_{0}}\right) l_{j_{0}}+\left(4+a_{i_{s} j_{0}^{\prime}}\right) l_{j_{0}^{\prime}}}{(4 n-1)+\sum_{t=1}^{n} a_{i_{s} i_{t}}}
$$

has to be 0 . Let us prove this. Firstly, if $l_{j_{0}}=-l_{j_{0}^{\prime}}$, then

$$
|h|=\left|\frac{a_{i_{s} j_{0}}-a_{i_{s} j_{0}^{\prime}}}{(4 n-1)+\sum_{t=1}^{n} a_{i_{s} i_{t}}}\right|<\frac{\left|a_{i_{s} j_{0}}\right|+\left|a_{i_{s} j_{0}^{\prime}}\right|}{4 n-2}<\frac{1}{2}, n \geq 1 .
$$

Secondly, assume $l_{j_{0}}=l_{j_{0}^{\prime}}=1$. then

$$
|h|=\left|\frac{8+a_{i_{s} j_{0}}+a_{i_{s} j_{0}^{\prime}}}{(4 n-1)+\sum_{t=1}^{n} a_{i_{s} i_{t}}}\right|<\frac{8+\left|a_{i_{s} j_{0}}\right|+\left|a_{i_{s} j_{0}^{\prime}}\right|}{4 n-2}<\frac{9}{10}, n \geq 3 .
$$

It follows that $h=0$, when $n \geq 3$. If $n=1$, with (21) we have

$$
3 h-8=a_{i_{s} j_{0}}+a_{i_{s} j_{0}^{\prime}}-h \sum_{t=1}^{n} a_{i_{s} i_{t}} \stackrel{h=3}{\leq}\left|a_{i_{s} j_{0}}\right|+\left|a_{i_{s} j_{0}^{\prime}}\right|+3\left|\sum_{t=1}^{n} a_{i_{s} i_{t}}\right|<1,
$$

It follows that $h \neq 3$. If $h=3+h_{0}$, with (21) we have

$$
3 h_{0}+1=a_{i_{s} j_{0}}+a_{i_{s} j_{0}^{\prime}}-3 \sum_{t=1}^{n} a_{i_{s} i_{t}}-h_{0} \sum_{t=1}^{n} a_{i_{s} i_{t}},
$$

and

$$
\left|h_{0}\right|=\left|\frac{a_{i_{s} j_{0}}+a_{i_{s} j_{0}^{\prime}}-3 \sum_{t=1}^{n} a_{i_{s} i_{t}}-1}{3+\sum_{t=1}^{n} a_{i_{s} i_{t}}}\right|<\frac{1+1}{2}=1 .
$$

So there is no non-zero $h$ satisfying (21). If $n=2$,

$$
8-7 h=-a_{i_{s} j_{0}}-a_{i_{s} j_{0}^{\prime}}+h \sum_{t=1}^{n} a_{i_{s} i_{t}} \stackrel{h=1}{\leq}\left|a_{i_{s} j_{0}}\right|+\left|a_{i_{s} j_{0}^{\prime}}\right|+\left|\sum_{t=1}^{n} a_{i_{s} i_{t}}\right|<1 .
$$

Similarly, we can show that there is no non-zero $h$ satisfying (21) when $n=1$. Thirdly, assume $l_{j_{0}}=1$ and $l_{j_{0}^{\prime}}=0$. With (21) we have

$$
|h|=\left|\frac{4+a_{i_{s} j_{0}}}{(4 n-1)+\sum_{t=1}^{n} a_{i_{s} i_{t}}}\right|<\frac{4+\left|a_{i_{s} j_{0}}\right|}{4 n-2}<\frac{5}{6}, n \geq 2 .
$$

If $n=1$, with (21) we have

$$
4-3 h=-a_{i_{s} j_{0}}+h \sum_{t=1}^{n} a_{i_{s} i_{t}} \stackrel{h=1}{\leq}\left|a_{i_{s} j_{0}}\right|+\left|\sum_{t=1}^{n} a_{i_{s} i_{t}}\right|<1,
$$

It follows that $h \neq 1$. If $h=1+h_{0}$, with (21) we have

$$
\left|h_{0}\right|=\left|\frac{1+a_{i_{s} j_{0}}-\sum_{t=1}^{n} a_{i_{s} i_{t}}}{3+\sum_{t=1}^{n} a_{i_{s} i_{t}}}\right|<\frac{1+1}{2}=1 .
$$

So we have no non-zero $h$ satisfying (21). Consequently, $h$ has to be 0 .

It follows from $h=0$ that $k=0$. Therefore

$$
\langle\alpha, k\rangle+\langle\beta, l\rangle=0+\lambda_{j_{0}} l_{j_{0}}+\lambda_{j_{0}^{\prime}} l_{j_{0}^{\prime}} \neq 0 .
$$


Then we complete the proof. Finally let us verify the assumption $\left|k_{s_{2}}-k_{s_{1}}\right|<1$. To this end, we need to estimate $\sum_{t=1}^{n}\left|k_{t}\right|$. With (19), we have

$$
\begin{aligned}
\sum_{s=1}^{n}\left|k_{s}\right| & =\sum_{s=1}^{n}\left|\sum_{t=1}^{n}\left(4+a_{i_{s} i_{t}}\right) k_{t}+\left(4+a_{i_{s} j_{0}}\right) l_{j_{0}}+\left(4+a_{i_{s} j_{0}^{\prime}}\right) l_{j_{0}^{\prime}}\right| \\
& \leq \sum_{s=1}^{n}\left(4\left|\sum_{t=1}^{n} k_{t}\right|+\sum_{t=1}^{n}\left|a_{i_{s} i_{t}} k_{t}\right|+\left|4+a_{i_{s} j_{0}}\right|+\left|4+a_{i_{s} j_{0}^{\prime}}\right|\right) \\
& <4 n\left|\sum_{t=1}^{n} k_{t}\right|+\frac{n b}{M^{2}} \sum_{t=1}^{n}\left|k_{t}\right|+\left(8+\frac{2 b}{M}\right) n .
\end{aligned}
$$

Sum up the equations (19) from $s=1$ to $n$, we have

$$
(4 n-1) \sum_{t=1}^{n} k_{t}+\sum_{s=1}^{n} \sum_{t=1}^{n} a_{i_{s} i_{t}} k_{t}+\sum_{s=1}^{n}\left(\left(4+a_{i_{s} j_{0}}\right) l_{j_{0}}+\left(4+a_{i_{s} j_{0}^{\prime}}\right) l_{j_{0}^{\prime}}\right)=0,
$$

and

$$
\begin{aligned}
(4 n-1)\left|\sum_{t=1}^{n} k_{t}\right| & \leq\left|\sum_{s=1}^{n} \sum_{t=1}^{n} a_{i_{s} i_{t}} k_{t}\right|+\left|\sum_{s=1}^{n}\left(4+a_{i_{s} j_{0}}\right)\right|+\left|\sum_{s=1}^{n}\left(4+a_{i_{s} j_{0}^{\prime}}\right)\right| \\
& \leq \sum_{s=1}^{n} \sum_{t=1}^{n}\left|a_{i_{s} i_{t}} k_{t}\right|+\left(8+\frac{2 b}{M}\right) n \\
& \leq \frac{n b}{M^{2}} \sum_{t=1}^{n}\left|k_{t}\right|+\left(8+\frac{2 b}{M}\right) n .
\end{aligned}
$$

Thus

$$
\sum_{s=1}^{n}\left|k_{s}\right|<\frac{8 n-1}{4 n-1} \frac{n b}{M^{2}} \sum_{t=1}^{n}\left|k_{t}\right|+\frac{8 n-1}{4 n-1}\left(8+\frac{2 b}{M}\right) n
$$

and

$$
\sum_{t=1}^{n}\left|k_{t}\right|<\frac{\left(24+\frac{6 b}{M}\right) n}{1-\frac{3 n b}{M^{2}}}=\frac{\left(24+\frac{6 b}{M}\right) n}{M^{2}-3 n b} M^{2} .
$$

If $M$ satisfy $M^{2}-3 n b>100 n b$ and $M>8 b$, then $\sum_{t=1}^{n}\left|k_{t}\right|<\frac{M^{2}}{4 b}$. So $\left|k_{s_{2}}-k_{s_{1}}\right|<\frac{1}{2}+\frac{1}{2}<1$. This completes the proof of Lemma 6.1 .

Since $\lambda_{j}=j^{2}+\frac{\bar{V}}{j^{2}}+O\left(\frac{1}{j^{3}}\right)$, the spectral sequence $\beta$ satisfies condition B with $d=2$. It is clear that $\mathbf{X}_{Q}, \mathbf{X}_{R} \in \mathcal{A}\left(\ell^{a, p}, \ell^{a, p}\right)$ where $Q=\bar{G}$ and $|R|=$ $O\left(\|\hat{q}\|_{a, p}^{4}\right)+O\left(\|q\|_{a, p}^{6}\right)$. Therefore the condition $\mathrm{C}$ is satisfied with $g=6>4$. Finally Theorem 2 applies. Using Theorem 2, we finish the proof of Theorem 1.

\section{References}

[1] L. Chierchia, and J.You: KAM tori for $1 D$ nonlinear wave equations with periodic boundary condition, Comm. Math. Phys. 211, 497-525 (2000)

[2] S. B. Kuksin, Hamiltonian perturbations of infinite-dimensional linear systems with an imaginary spectrum, Funct. Anal. Appl., 21 (1987), 192-205

[3] S. B. Kuksin: Nearly integrable infinite-dimensional Hamiltonian system, Lecture Notes in Math, 1556, Springer-Verlag, New York, 1993

[4] S. B. Kuksin, and J.Pöschel: Invariant Cantor manifolds of quasi-periodic oscillations for a nonlinear Schrödinger equation, Ann. Math. 143, 149-179 (1996)

[5] J. Pöschel: Quasi-periodic solution for nonlinear wave equation, Comment. Math. Helvetici 71, 269-296 (1996) 
[6] J. Pöschel, and E.Trubowitz: Inverse Spectral Theory, Academic Press, Boston, 1987.

[7] E.C.Titchmarsh: eigenfunction expansions associated with second-order differential equations, Part I, Oxford: Clarendon Press 1962

[8] C. E. Wayne, Periodic and quasi-periodic solutions of nonlinear wave equations via KAM theory, Commun. Math. Phys.127 479-528 (1990)

[9] X. Yuan: Invariant tori of nonlinear wave equation with a given potential, Discrete and Continuous Dynamical Systems (in line for publication)

School of Mathematical Science, Fudan University, Shanghai 200433, China

School of Mathematical Science, Fudan University, Shanghai 200433, China

E-mail address: xpyuan@fudan.edu.cn 